\title{
Measuring mitochondrial respiration in adherent cells infected with Trypanosoma cruzi Chagas, 1909 using Seahorse extracellular flux analyser
}

\author{
Laura Maria González-Ortiz*, Juana Patricia Sánchez-Villamil*, Mike A. Celis-Rodríguez, Giovanni Lineros, \\ Sandra Sanabria-Barrera, Norma C. Serrano, Melvin Y. Rincon, Paula K. Bautista-Nino
}

Traslational Biomedical Research Group, Centro de Investigaciones, Fundación Cardiovascular de Colombia, Santander, Colombia.

*These authors contributed equally to this work

\begin{abstract}
Infection with Trypanosoma cruzi Chagas, 1909 is reported to increase the production of reactive oxygen species in patients with Chagas disease. Mitochondria dysfunction, host inflammatory response and inadequate antioxidant response are described as the main factors leading to oxidative stress during acute and chronic stages of the disease. The Seahorse XFe24 extracellular flux platform allows energy metabolism determination through mitochondrial respiration and glycolysis measurements. XFe 24 platform can be used in in vitro models of T. cruzi-infected cells, which allow the assessment and even modulation of endogenous conditions of infected cells, generating readouts of real-time cellular bioenergetics changes. In this protocol, we standardised the use of XFe24 technology in T. cruzi infected AC16 cardiomyocytes and SGHPL-5 trophoblasts. In addition, we provide a list of optimised assay specifications, advantages and critical steps to be considered during the process. Cardiomyocytes and trophoblasts are attractive target cells to evaluate the metabolic environment in acute, chronic and congenital Chagas transmission scenarios.
\end{abstract}

Keywords: Chagas disease, trophoblastic cells, cardiomyocytes, cellular respiration, mitochondrial bioenergetics.

Infection with the kinetoplastid Trypanosoma cruzi Chagas, 1909 is transmitted to humans predominantly by insect vectors, which deposit infective trypomastigotes with the faeces during blood meals. Once inside human host cells, $T$. cruzi trypomastigotes transform into dividing intracellular forms called amastigotes. Amastigotes duplicate inside of the host cell, transform to trypomastigotes which are released into the environment upon the death of the host cell. These trypomastigotes can either infect other healthy cells and transform again into amastigotes or they can be ingested by blood-sucking insect vectors.

Due to the intracellular nature of the parasite, in vitro models of T. cruzi infection have become useful tools for studies of the pathogenesis of Chagas disease, including the evaluation of the infection and metabolic cellular processes, parasite-host interaction, signaling perturbations and selection of potential therapeutic targets (Duran-Rehbein et al. 2014). Interestingly, the pathophysiology of Chagas disease can be evaluated in a wide variety of cell lines available in public and private repository banks.

In different rmammalian cellular culture models of $T$. cruzi infection, the most commonly used cell lines have been Vero (African Green monkey renal epithelial cells), LLC-MK2 (Rhesus monkey kidney epithelial cells) and peripheral blood mononuclear cells (PBMCs) (Duran-Rehbein et al. 2014). Additionally, other cell types, which were evaluated as models in T. cruzi infection include the human cardiomyocyte cells AC16 (Wen et al. 2018), primary cardiac fibroblasts (Aridgides et al. 2013) and human smooth muscle cells (HSMCs) (Andrade et al. 2012).

Although exposure to the infected faeces of the blood-sucking triatomine bug is the predominant form of $T$. cruzi infection, the parasite can also be transmitted from mothers to the foetus during pregnancy. Congenital transmission constitutes to be an important public health problem, with significant contribution to the spread of Chagas disease to non-endemic areas of Latin America, United States of America and Europe (Coura and Vinas 2010).

Different approaches have been developed to study the pathology of congenital T. cruzi infection, including the use of cytotrophoblastic cells (Diaz-Lujan et al. 2016), human placental chorionic villous explants (Castillo et al. 2017) and human placenta choriocarcinoma BeWo cells (Liempi et al. 2014). These studies have shown that $T$. cruzi infection leads to increased pro- and anti-inflammatory cytokine secretion and to increased turnover of trophoblastic cells as a way to confer immune protection against the parasite (Castillo et al. 2017). 


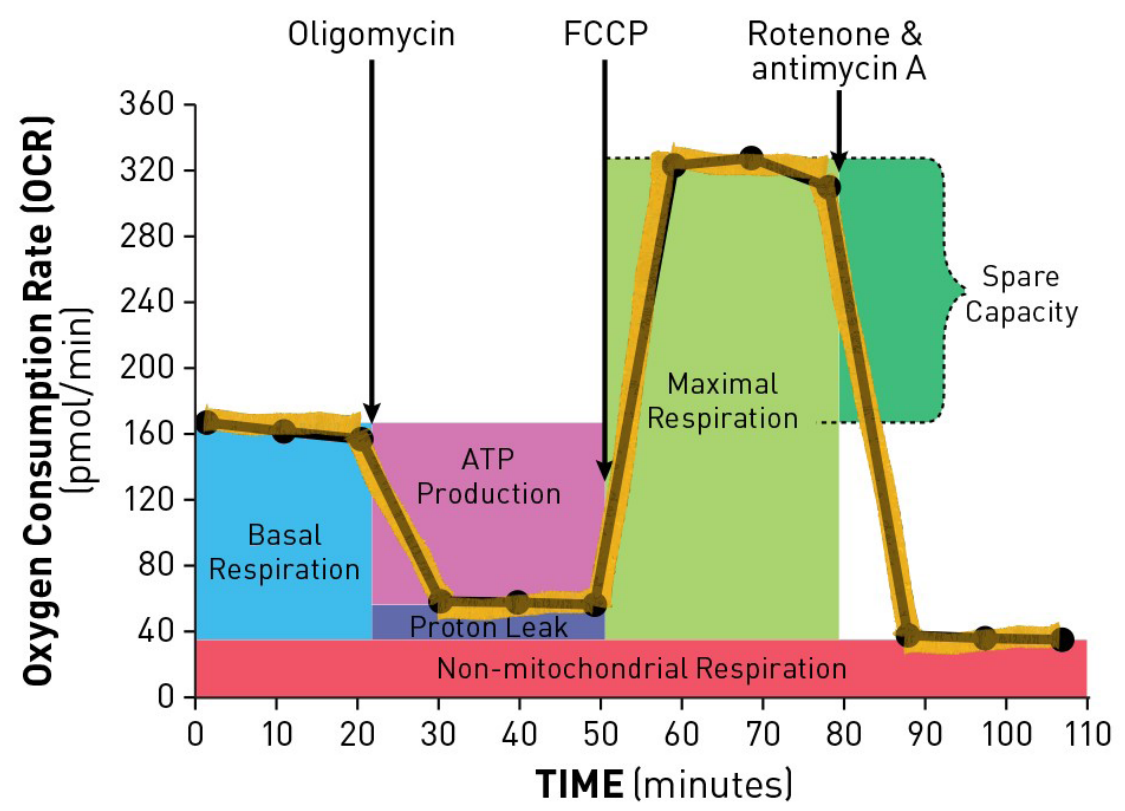

Fig. 1. Outline of extracellular flux assay and mitochondrial parameters evaluated. Taken from Agilent Seahorse XF Cell Mito Stress Test Kit, User Guide.

Reports from different countries indicate that up to $28 \%$ of infected mothers transmit the T. cruzi parasite to their foetuses, raising questions about the role of the placenta as a barrier to transmission (Cucunuba et al. 2012). In this context, understanding the early stages of $T$. cruzi infection, the complex interaction between parasite, immunometabolic responses of the mother, foetal and placental factors are significant. Trophoblast and stromal cells are the main cells present in human placenta (Gude et al. 2004). Trophoblast cells, in the intervillous space, constitute the first barrier that is in contact with the parasite T. cruzi (Benirschke et al. 2006). Additionally, cell lines obtained from first trimester placenta such as HTR-8/SVneo and SGHPL-5 (Saint Georges Hospital Placental cell line-5) (Choy et al. 2000) are the best option to study extravillous trophoblastic functions. Therefore, the study of metabolic phenotypes and mitochondrial function of trophoblast cells during acute Chagas infection could reveal valuable information about their response to T. cruzi.

The Seahorse XFe24 extracellular flux analyser (www. seahorsebio.com) measures the oxygen consumption rate (OCR) and the extracellular acidification rate (ECAR) of live cells allowing quantification of mitochondrial respiration and cellular glycolytic function. Measurements with a Seahorse XFe24 extracellular flux analyser require a small amount of cells and a short time to obtain results. The Cell Mito Stress Test is a standard assay for the evaluation of mitochondrial function. During the experiment, cells are challenged by the addition of four compounds (oligomycin, carbonyl cyanide-4-(trifluoromethoxy)phenylhydrazone (FCCP), antimycin and rotenone) that change the bioenergetic profile of the cell. Parameters calculated include mitochondrial basal respiration, ATP production, proton leak, maximum respiration and spare respiratory capacity. Figure 1 shows the outline of the Cell Mito Stress Test and the parameters calculated.
The test begins with a basal measurement of OCR, followed by oligomycin injection, which, by inhibiting complex V, rapidly hyperpolarises the mitochondrial membrane, preventing the pumping of protons through the complexes. This hyperpolarised state is reversed by the injection of the uncoupling agent FCCP, and in response the cells increase the speed of electron transport to try to recover the membrane potential, maximising the OCR. Finally, the injection of $\mathrm{A} / \mathrm{R}$ to inhibit mitochondrial complexes III and I completely stops mitochondrial respiration, and this is reflected in OCR readings of nearly zero.

Considering the advantages of this technology and the important and particular need to study cellular responses to T. cruzi infection, we describe a protocol to study T. cru$z i$-induced metabolic changes in trophoblastic cells. The aim of the current work is to provide a tool for measuring cellular bioenergetics in adherent cells infected with $T$. cru$z i$ using a Seahorse XFe24 instrument.

\section{MATERIALS AND METHODS}

\section{Materials and reagents}

- Ham's F-10 medium, DMEM F-12 50/50 Mix, REF \#10-070CVR by Corning Inc. (Corning, NY, USA).

- RPMI 1640, REF \#50-020-PBR by Corning Inc.

- DMEM / F-12 50/50, REF \#90-091-PBR by Corning Inc.

- DMEM, REF \#DMP52-50LT by Caisson (Smithfield, UT, USA).

- Fetal bovine serum (FBS) and Penicillin/Streptomycin (P/S) (Shah-Simpson et al. 2016) by Corning Inc., Product Code \#35-015-CV.

- Trypsin-EDTA $0.125 \%$, CAT \# 15400-054 by Gibco - Life Technologies (Waltham, MA, USA).

- PBS without $\mathrm{Ca} 2+$ and $\mathrm{Mg} 2+$ by Gibco - Life Technologies.

- Seahorse XF Calibrant solution, part number 100840-000 by Agilent (Santa Clara, CA, USA). 

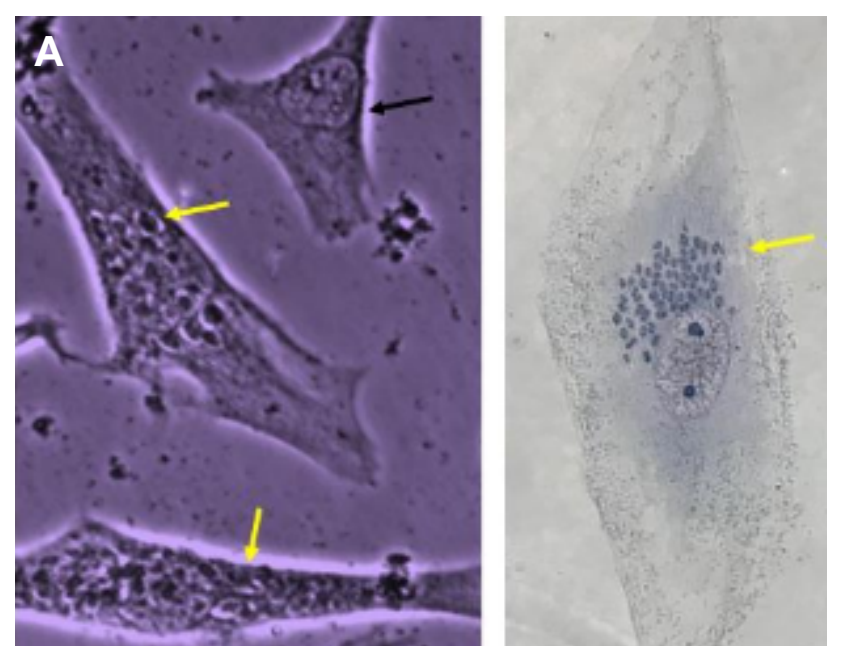

B
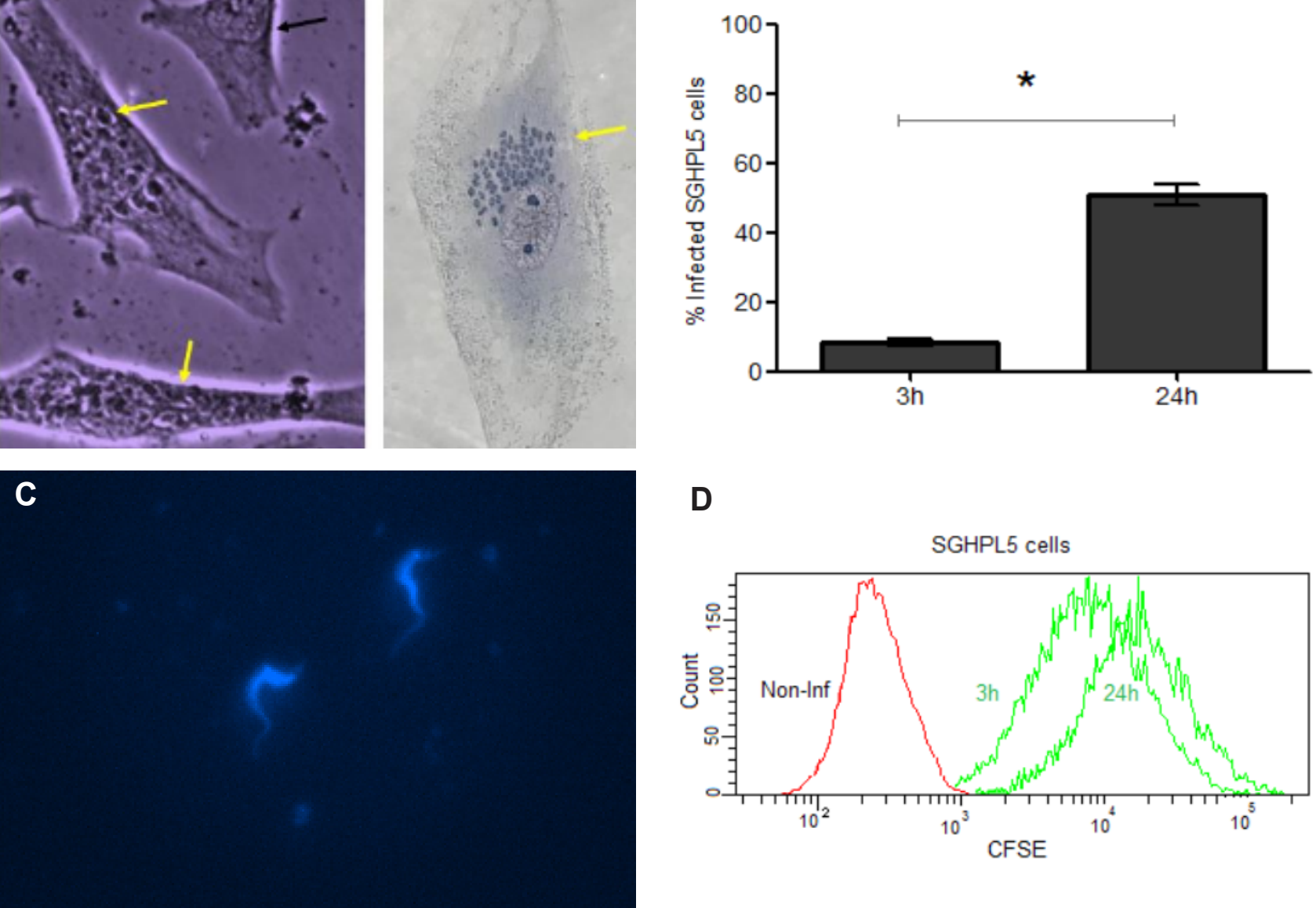

D

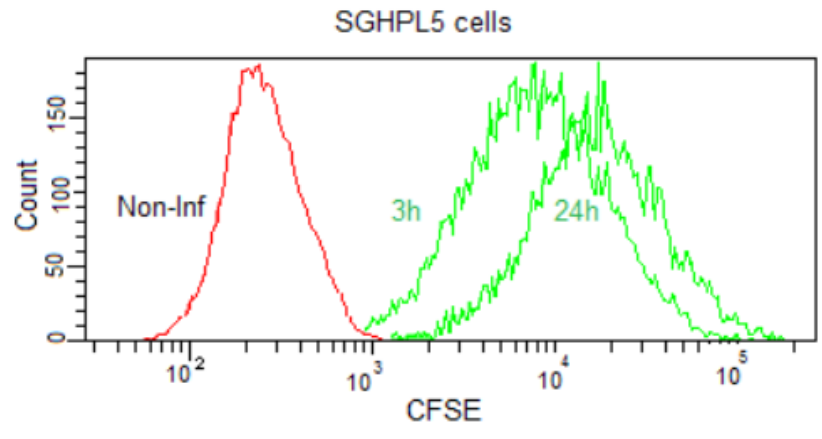

Fig. 2. Monitoring of infection with Trypanosoma cruzi Chagas, 1909 in SGHPL-5 cells. A - Giemsa staining shows intracellular forms of T. cruzi (yellow arrows) after 24h of infection vs a non-infected cell (black arrow); B - Percentage of infected cells after 3 and $24 \mathrm{~h}$ of infection at $1: 5$ cell/ parasite ratio; data are presented as means $\pm \mathrm{SD}$ (4 independent experiments); $* P<0.05$ (Chi-square test); C-CFSE-stained parasites were used for in vitro infection of SGHPL-5 cells at $1: 5$ cell/ parasite ratio and the fluorescence emitted from intracellular parasites was evaluated by flow cytometry; D - Representative histogram plot showing CFSE fluorescence by cells after 3 and $24 \mathrm{~h}$ of infection with $T$. cruzi.

- $1 \mathrm{~N} \mathrm{NaOH}, \mathrm{CAT}$ \#AA35629K2 by Alfa Aeser (Haverhill, MA, USA).

- Oligomycin*, Product Code 11342 by Cayman Chemical Company (Ann Arbor, MI, USA).

- Carbonyl cyanide-4-(trifluoromethoxy) phenylhydrazone (FCCP)*, CAT \#sc-203578 by Santa Cruz Biotechnology, Inc. (Dallas, TX, USA).

- Antimycin A*, Product Code \#19433 by Cayman Chemical Company.

- Rotenone*, Product Code \#13995 by Cayman Chemical Company.

- Carboxyfluorescein diacetate succinimidyl ester (CFSE), CAT \# 423801 by Biolegend, (San Diego, CA, USA).

*Oligomycin, FCCP, antimycin A and rotenone were initially reconstituted in dimethyl sulfoxide (DMSO) and then diluted to the final concentrations required for the assay in DMEM basal assay medium.

\section{Supplies and equipment}

- Sterile plastic material manufactured by Corning Inc.

- XFe24 FluxPak, part number 102340-100 (Seahorse Bioscience: including sensor cartridges and cell culture microplates)
- CO2 Incubator - Sanyo 19AIC CO2

- Non-CO2 incubator - VWR 12620-910 Incubating Rocker

- Microbiological safety cabinet at appropriate containment level - CSB Clase II Tipo A1 AIRR100

- Water bath set to appropriate temperature - Microprocessor Controlled 280 series Water Bath Model 2853 Thermo Scientific

- Inverted phase contrast microscope - Eclipse Ti-S, Nikon Instruments Inc.

- Centrifuge - Thermo Scientific Sorvall BioFuge Stratos Refrigerated High-Speed Benchtop Centrifuge

- Haemocytometer/ Neubauer chamber

Remarks. For the following procedures, an aseptic technique should be followed at all times. All the solutions and equipment coming into contact with cells must be sterile. Biosafety practices must be followed according to local and international regulations.

\section{Cell lines}

- SGHPL-5 cells were grown in Ham's F-10 medium containing $10 \% \mathrm{FBS}$ and $1 \% \mathrm{P} / \mathrm{S}$.

- AC16 cells were grown in DMEM F-12 50/50 Mix 12.5\% FBS and $1 \% \mathrm{P} / \mathrm{S}$. 

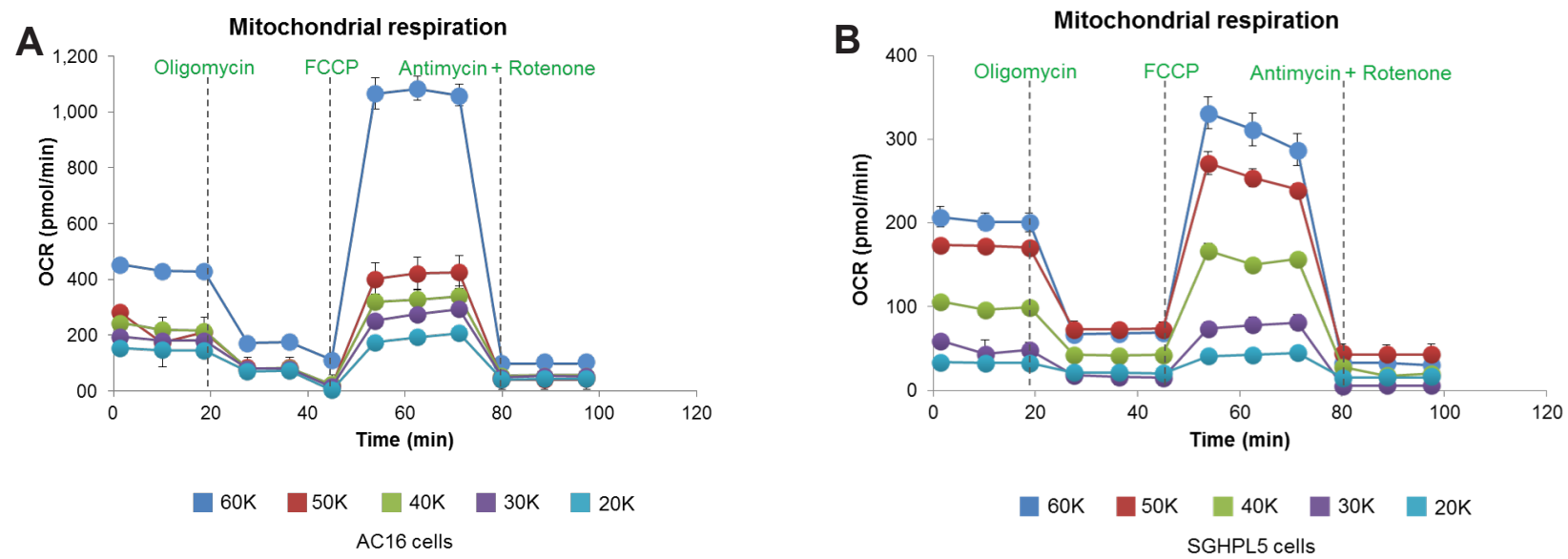

Fig. 3. Oxygen consumption rate responses in cells seeded at different densities. A-OCR responses in AC16 cells; B - OCR responses in SGHPL-5 cells. In both cell lines OCR was measured under basal conditions followed by sequential addition of oligomycin (1.5 $\mu \mathrm{M})$, FCCP $(1.5 \mu \mathrm{M})$ and antimycin $\mathrm{A} /$ rotenone $(\mathrm{A} / \mathrm{R})(3 \mu \mathrm{M}) . \mathrm{K}=10^{3}$ cells per well. OCR values were normalised to cell protein. Each curve is a representative from three independent experiments; four technical replicates were mounted within each experiment.

- $\mathrm{C} 2 \mathrm{C} 12$ cells were grown in RPMI medium supplemented with $10 \%$ FBS and $1 \%$ P/S. However, when infected with Trypanosoma cruzi trypomastigotes, $\mathrm{C} 2 \mathrm{C} 12$ cells were grown in RPMI medium supplemented with $5 \% \mathrm{FBS}$ and $1 \% \mathrm{P} / \mathrm{S}$.

- Trypanosoma cruzi strain SilvioX10/4 was donated for research purposes to the Traslational Biomedical Research Group, Fundación Cardiovascular de Colombia, Santander, Colombia (courtesy of Garg N., Department of Microbiology and Immunology, University of Texas Medical Branch, Galveston, TX 77555, USA). Parasites were grown in RPMI medium with $5 \% \mathrm{FBS}$ and $1 \% \mathrm{P} / \mathrm{S}$.

- For running the assay on the XFe24 extracellular flux analyser, the medium has to be replaced with assay medium. That is DMEM-based with varying concentrations of glucose, pyruvate and L-glutamine depending on the cell line. It must not contain sodium bicarbonate and should be prepared only on the assay day. For the AC16 cell line the assay medium contained $17.48 \mathrm{mM}$ glucose, $1.25 \mathrm{mM}$ pyruvate and $2.5 \mathrm{mM}$ L-glutamine. For the SGHPL-5 cell line the assay medium contained $6.1 \mathrm{mM}$ glucose, $1.0 \mathrm{mM}$ pyruvate and $1.25 \mathrm{mM}$ L-glutamine (Ba et al. 2010, Kipkeew et al. 2016).

\section{EXPERIMENTAL PROCEDURE}

\section{Parasite culture, cell infection process and monitoring of the infection process}

\subsection{Parasite culture and cell infection process}

Before infecting the cells under study, it is necessary to harvest sufficient numbers of trypomastigotes. $\mathrm{C} 2 \mathrm{C} 12$ cells were used for replication of the parasite. The steps to achieve infection of $\mathrm{C} 2 \mathrm{C} 12$ cells are listed below.

1. Plate $\mathrm{C} 2 \mathrm{C} 12$ cells in sterile flasks at $30 \%$ confluence. Use RPMI medium supplemented with $5 \%$ FBS and $1 \% \mathrm{P} / \mathrm{S}$.

2. Infect the $\mathrm{C} 2 \mathrm{C} 12$ cells with parasites at a minimum cell: parasite ratio of $1: 2$ and incubate at $37^{\circ} \mathrm{C}$ with $5 \% \mathrm{CO} 2$. The success of cell infection with $T$. cruzi can be easily verified by examining the flasks under inverted microscope for the presence of intracellular amastigotes.
3. After 24 hours remove the medium and wash with prewarmed PBS once. Replace with new medium and place in incubator at $37^{\circ} \mathrm{C}$ with $5 \% \mathrm{CO} 2$.

4. Grow the cells for 4-5 days; change the medium when there is a change in colour of the $\mathrm{pH}$ indicator.

5. After growing the cells for 4-5 days there should be enough trypomastigotes in suspension. This is the ideal time to start harvesting the trypomastigotes in the supernatant. Trypomastigotes can be harvested from the same flask for several days. However, the culture should be discarded when extracellular amastigotes represent approximately $20-40 \%$ of the visual field because it is difficult to separate amastigotes from trypomastigotes by centrifugation.

6. Collect the supernatant in a conical centrifuge tube.

7. Centrifuge for ten minutes at $1,000 \mathrm{rpm}$ to remove $\mathrm{C} 2 \mathrm{C} 12$ cells and cellular detritus.

8. Transfer the supernatant to a new tube and centrifuge for 10 minutes at $4,000 \mathrm{rpm}$ to concentrate the parasites.

9. Discard the supernatant and gently resuspend the pellet in fresh low FBS medium. Use the Neubauer chamber to establish the concentration of trypomastigotes. This trypomastigote suspension can be used to infect the cells of interest, in our case AC16 and SGHPL-5 cells.

10. Add an appropriate volume of the parasite suspension to a new flask to start a new cell culture infection or adjust the volume for Seahorse assay (see below: 3.1.2 T. cruzi-infection treatment).

Remarks. Trypanosoma cruzi is classified as a biosafety level 2 microorganism and all assays should be performed using personal protection equipment in a Class II biological safety hood. Trypanosoma cruzi is a highly infectious protozoan parasite and before following this protocol it is important to receive training and wear appropriate protective clothing.

\subsection{Monitoring of T. cruzi-cell infection}

To monitor T. cruzi cell infection, Giemsa and CFSE staining were carried out. AC16 and SGHPL-5 cells were seeded in 24well plates at $2 \times 10^{3}$ cells per well and exposed to trypomastigote suspension, under the conditions described above. For Giemsa 

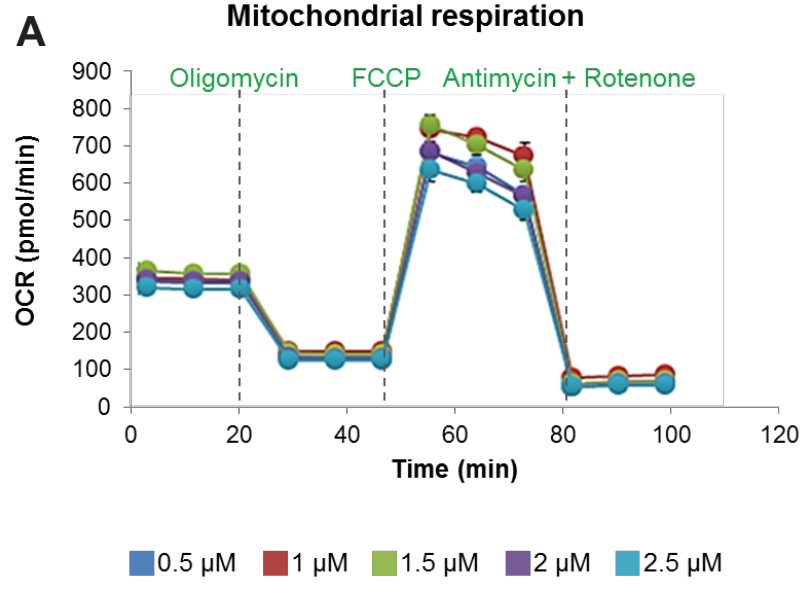

AC16 cells

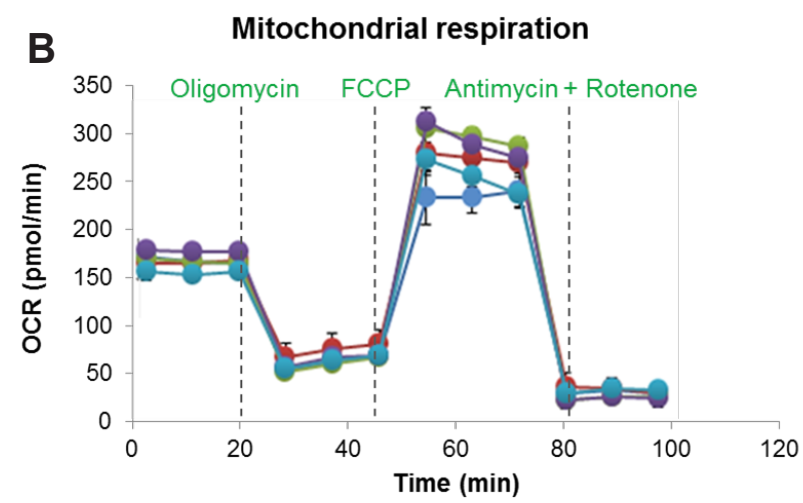

$0.5 \mu \mathrm{M} \quad \square 1 \mu \mathrm{M} \quad 1.5 \mu \mathrm{M} \quad \square 2 \mu \mathrm{M} \quad 2.5 \mu \mathrm{M}$

SGHPL5 cells

Fig. 4. Optimisation of FCCP concentrations. A - Oxygen consumption rate (OCR) trace in $\mathrm{AC} 16\left(50 \times 10^{3}\right.$ cells/well $)$; $\mathbf{B}-\mathrm{OCR}$ trace in SHGPL-5 $\left(60 \times 10^{3}\right.$ cells/well). The assays were run under basal conditions followed by addition of oligomycin $(2 \mu \mathrm{M})$, FCCP $(0.5$ $\mu \mathrm{M}, 1 \mu \mathrm{M}, 1.5 \mu \mathrm{M}, 2 \mu \mathrm{M}$ and $2.5 \mu \mathrm{M})$ and $\mathrm{A} / \mathrm{R}(3 \mu \mathrm{M})$. OCR values were normalised to cell protein. Each curve is a representative from three independent experiments; four technical replicates were mounted within each experiment.

staining: after 3- and 24-hours-exposure of the cells to the trypomastigote suspension, each well was washed with PBS to ensure total removal of extracellular parasites and fresh low FBS medium was added. Plates were incubated for 2 days to ensure formation of intracellular amastigotes. The medium was then removed and each of the wells was fixed with methanol for 15 minutes, followed by $10 \%$ Giemsa staining (v/v) for 30 minutes. The percentage of cells with intracellular amastigotes was quantified under the microscope. Figure 2A shows a representative image of SGHPL-5 cells with intracellular amastigote nests. After 3 hours exposure to trypomastigotes, less than $10 \%$ of the cells were infected, while after 24 hours of exposure $50 \%$ of the cells were infected (Fig. 2B).

For CFSE staining: trypomastigotes were resuspended in $1 \mathrm{ml}$ of PBS with $5 \mu \mathrm{M}$ of CFSE and incubated for 10 minutes in the dark at $37^{\circ} \mathrm{C}$. After this, they were washed twice with $5 \mathrm{ml}$ of tempered culture medium and used to infect the cells. The viability of the CFSE-stained T. cruzi parasites was tested by observing mobility under a light microscope and confirmed by observation under a fluorescence microscope (Eclipse Ti-S, Nikon Instruments Inc.). Figure $2 \mathrm{C}$ shows two CFSE-stained trypomastigotes. After 3 and 24 hours of exposure to CFSE-stained trypomastigotes, culture plates were washed as described for Giemsa staining. Cells were incubated for two days and then suspended in PBS containing $0.1 \%$ sodium azide and $2 \%$ FBS and run on an analytical flow cytometer BD FACS Canto II with laser emission at $519 \mathrm{~nm}$. The results of CFSE expression are presented in Fig. 2D. In agreement with the Giemsa staining observations, there were more cells infected after 24 hours of parasite exposure.

\section{Standardisation of basic parameters for setting up an assay in the Seahorse XFe24 extracellular flux analyser}

\subsection{Cell seeding density assay}

For cell seeding density assays in the Seahorse XFe24 extracellular flux analyser it is necessary to have cell culture dishes at $60-90 \%$ confluence to assure a dynamic metabolic cell rate. Cells harvested from these dishes are seeded in 24-well Seahorse culture microplates where the seeding surface area of each well is about $200 \mathrm{~mm}^{2}$. Cell density was optimised by plating five different cell densities: $6,5,4,3$ and $2 \times 10^{4}$ cells/well for the SGHPL-5 cell line and 5, 4, 3, 2 and $1 \times 10^{4}$ cells/well for the AC16 cell line.

The criteria used to select the optimal cell density were: (i) visual examination under a microscope looking for the density in which the monolayer cells are seeded at $60-80 \%$ confluence; (ii) basal OCR readings between $50-400 \mathrm{pmol} / \mathrm{min}$ obtained in the Seahorse instrument. Figure 3 shows representative results out of 3 independent experiments obtained for each cell line. As observed in Fig. 3A there is a higher increase in maximal respiration at $5 \times 10^{4}$ cells/well in AC16 cells, even if basal OCR is slightly above $400 \mathrm{pmol} / \mathrm{min}$. Therefore, the optimal cell density for AC16 cells was $5 \times 10^{4}$ cells/well. In Fig. $3 \mathrm{~B}$ it is seen that as cell density increases, the maximal respiration is also gradually increased. Careful study of each specific cell line should be considered due to possible cell detachment in wells after infection with $T$. cruzi. The possibility of cell detachment depends on time elapsed after infection and possibly on the strain of parasite.

\subsection{FCCP optimisation assay}

Once the optimal cellular density has been selected, the next step is to define the concentration of FCCP necessary to achieve total mitochondrial uncoupling in order to determine the maximal oxygen consumption that cells can sustain. FCCP was prepared initially in DMSO (to a concentration of $100 \mathrm{mM}$ ) and then diluted to its final concentration in DMEM basal assay medium. Final FCCP concentrations per well of $0.5 \mu \mathrm{M}, 1 \mu \mathrm{M}, 1.5 \mu \mathrm{M}, 2$ $\mu \mathrm{M}$ and $2.5 \mu \mathrm{M}$ were tested for both cell lines. For AC16 cells, the concentration of FCCP $1.5 \mu \mathrm{M}$ was chosen, corresponding to the highest OCR reading after the injection of FCCP (Fig. 4A); while for SGHPL-5 cells the optimal FCCP concentration was 1.0 $\mu \mathrm{M}$ (Fig. 4B). In both cell lines, the highest tested concentration of FCCP $(2.5 \mu \mathrm{M})$ resulted in lower OCR compared to the other concentrations, possibly because the system reached its limit of maximal capacity of oxygen consumption or because at this concentration FCCP induces mitochondria damage. 

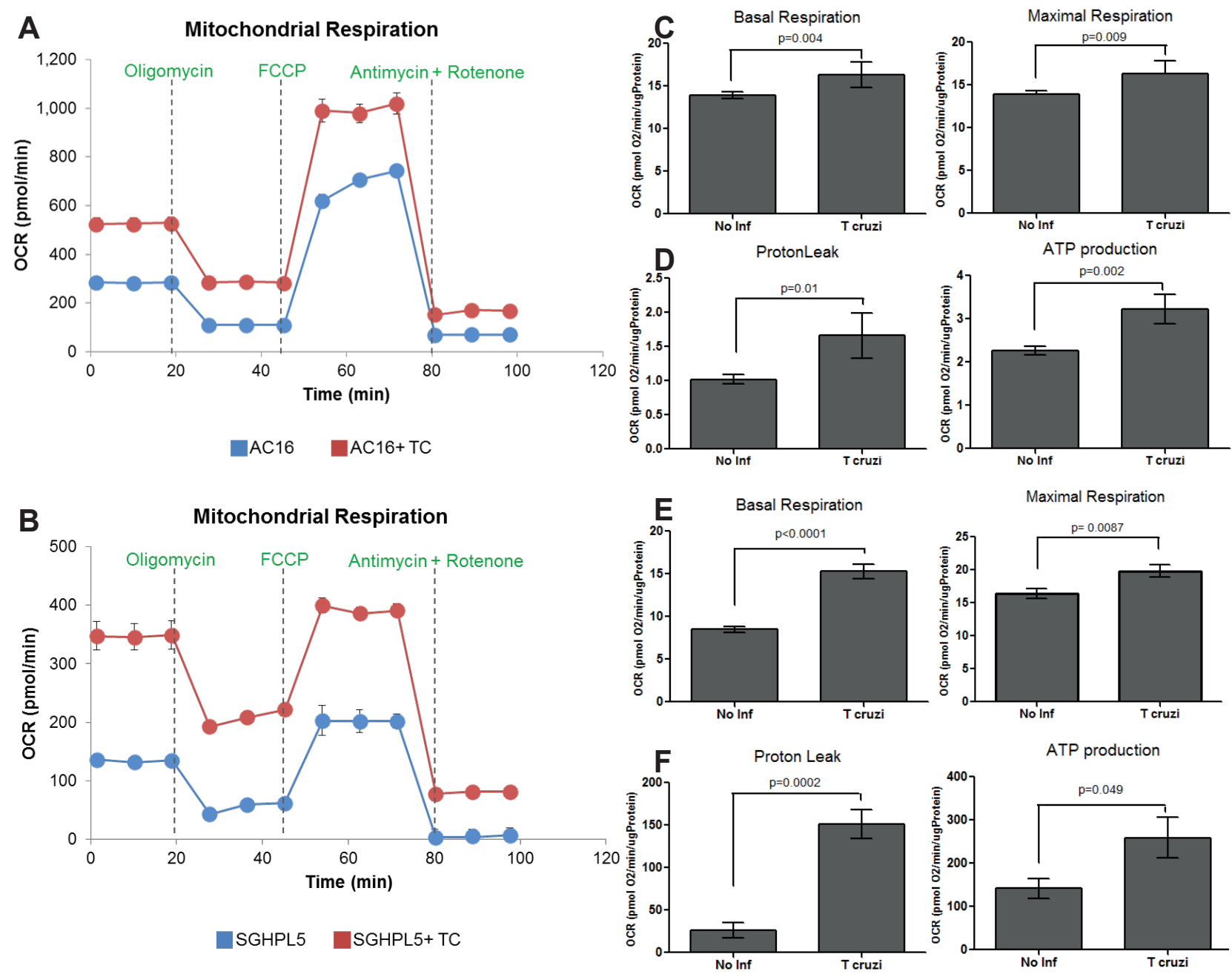

Fig. 5. Mitochondrial function parameters of AC16 and SGHPL-5 cells infected with Trypanosoma cruzi Chagas, 1909 compared with non-infected cells using XFe24 analyser. Oxygen consumption rate (OCR) traces under basal conditions in AC16 (A) and SGHPL-5 cells (D). Red line shows traces of infected cells, and blue line shows traces of non-infected cells. Basal and Maximal OCR from non-infected and T. cruzi-infected AC16 (B) and SGHPL-5 cells (E). Proton leak and ATP production in non-infected and T. cruzi-infected AC16 (C) and SGHPL-5 cells (F). Data are presented as mean \pm SEM of normalised values of different oxygen consumption rates (OCRs), $\mathrm{n}=4$ per group. Student's $t$ test was used to compare groups.

3. Procedure for the Mitochondrial Stress Test on the XFe24 extracellular flux analyser in cells infected with trypomastigotes $T$. cruzi Silvio X10/4

\subsection{Day 1 of assay: plating cells and T. cruzi infection}

\subsubsection{Cell seeding}

1. Use an inverted microscope to assess cell confluence and the absence of microbiological contaminants in the dishes or flasks. Cell confluency should be between 60 and $90 \%$.

2. Remove the medium.

3. Wash the cell monolayer gently with pre-warmed PBS one or two times.

4. Add enough pre-warmed trypsin to cover the cell monolayer.

5. Place in incubator at $37^{\circ} \mathrm{C}$ with $5 \% \mathrm{CO} 2$ for approximately 5 minutes.

6. Examine the cells under microscope for detachment.

7. When the cells have detached, add pre-warmed medium, aspirate cells by gentle pipetting and transfer to a conical sterile tube.
8. Centrifuge for ten minutes at 1,000 rpm.

9. Discard the supernatant and resuspend the cell pellet with $2 \%$ FBS growth medium.

10. Count the cells and add the appropriate volume of medium to achieve the desired concentration of cells in suspension.

11. Seed $100 \mu \mathrm{l} /$ well of the optimal cell suspension in the Seahorse 24-well cell culture microplate. Please note that wells A1, B4, C3 and D6 should not be used. They are the background controls and should contain only medium.

12. Incubate at $37^{\circ} \mathrm{C}$ with $5 \% \mathrm{CO}_{2}$ for 2 hours or until the cells adhere to the bottom of the plate.

\subsubsection{T. cruzi infection treatment}

1. During incubation prepare the parasites according to the steps listed in section 1.1 Parasite culture and cell infection process and resuspend trypomastigotes in low FBS growth medium. Here we supplemented the medium with $2 \%$ FBS for both AC16 and SGHPL-5 cells.

2. After 2-hour incubation at $37^{\circ} \mathrm{C}$ with $5 \% \mathrm{CO}_{2}$, take the microplate out of the incubator and add $150 \mu \mathrm{l}$ of $2 \%$ FBS 

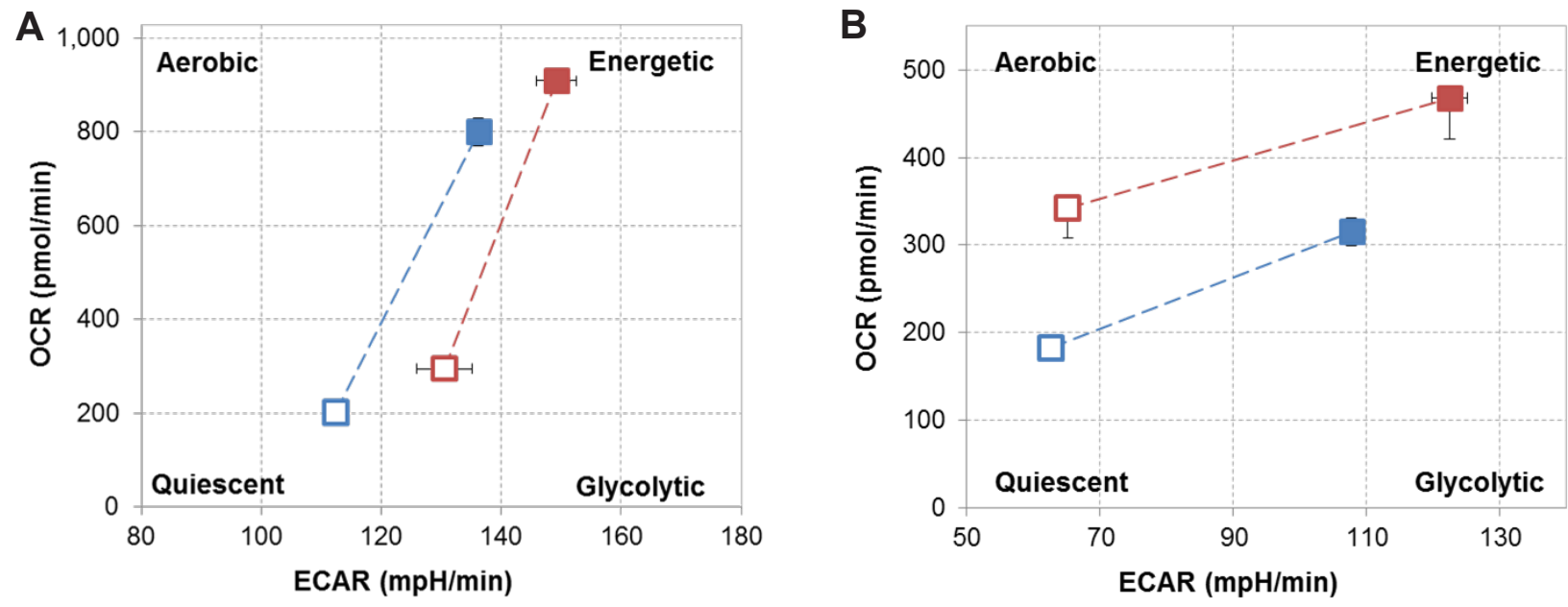

Fig. 6. Cell energy phenotype profile of AC16 and SGHPL-5 cells infected with Trypanosoma cruzi compared with non-infected cells using XFe24 analyser. Energetic phenotype map, basal and maximal OCR and extracellular acidification rates (ECAR), both in AC16 (A) and in SGHPL-5 cells (B). Data in blue corresponds to non-infected cells and data in orange corresponds to Trypanosoma cruzi infected cells, open symbols correspond to the baseline and closed symbols to the stressed condition.

growth medium to the control wells (non infected cells) and add $150 \mu \mathrm{l}$ of parasite suspension to the sample wells, keeping the $1: 5 \mathrm{cell} /$ parasite ratio.

3. Incubate at $37^{\circ} \mathrm{C}$ with $5 \% \mathrm{CO}_{2}$ for 24 hours or the defined time period for the experiments.

4. The Seahorse XFe24 Sensor Cartridge should be hydrated with the calibrant solution overnight at $37^{\circ} \mathrm{C}$ in a non- $\mathrm{CO}_{2}$ incubator For this purpose go to: https://www.agilent.com/ cs/library/usermanuals/public/XFe24_day_before_cartridge hydration.pdf

\subsection{Day 2 of assay: Run assay}

1. Verify the cells under the microscope to confirm cell health, seeding uniformity and absence of contamination.

2. Prepare DMEM basal assay medium and supplement with glucose, pyruvate and glutamine concentrations according to the requirements of each cell line.

3. Warm the assay medium to $37^{\circ} \mathrm{C}$ and then adjust $\mathrm{pH}$ to 7.3 7.4 with $0.1 \mathrm{~N} \mathrm{NaOH}$

4. Wash cells in the wells twice with the tempered assay medium (to $37^{\circ} \mathrm{C}$ ) to avoid cellular detachment*. The final volume of the assay medium in each well must be $525 \mu 1$ in order to reach a final assay volume of $600 \mu \mathrm{l}$ after addition of the first inhibitor, in this case oligomycin.

5. Incubate cell culture microplate at $37^{\circ} \mathrm{C}$ in a non- $\mathrm{CO}_{2}$ incubator for 30-60 minutes.

6. Use the mobile app "XF Dilution Calculator" to calculate and prepare (diluted with assay medium) the inhibitors oligomycin, FCCP and antimycin/rotenone $(\mathrm{A} / \mathrm{R})$ to inject a fixed volume of $75 \mu \mathrm{l}$ per well. In our case, we used final concentrations in each well of $2 \mu \mathrm{M}$ oligomycin, $1.5 \mu \mathrm{M} \mathrm{FCCP}$ and 3 $\mu \mathrm{M} \mathrm{A} / \mathrm{R}$ for experiments with AC16 cells. For SGHPL- 5 cells we used final concentrations in each well of $2 \mu \mathrm{M}$ oligomycin, $1.0 \mu \mathrm{M} \mathrm{FCCP}$ and $3 \mu \mathrm{M} \mathrm{A} / \mathrm{R}$.

7. Inject $75 \mu 1$ of each mitochondrial inhibitor solution to each injector port within a sensor cartridge: pipette $75 \mu$ of $8 \times$ oligomycin in Port A, $75 \mu 1$ of $9 \times$ FCCP in Port B and $75 \mu 1$ of $10 \times A / R$ in Port $C$ on top of the sensor cartridge.

8. Set up the Wave software and run the Seahorse standard configuration.

*It is of utmost importance to avoid contamination with parasites in the culture microplate. If besides infected cells, there are parasites in suspension in the microplate wells, the metabolic readings will be confounded (readings generated from parasites and cells). For this reason, we standardised the number of washing steps to 8 to guarantee complete removal of parasites.

\section{RESULTS}

Figure 5A,D show that infection with $T$. cruzi induces changes in mitochondrial respiration rates. In both cell lines, basal respiration in infected cells is increased. This could be due to the higher ATP demand caused by the infection condition (Fig. 5B, E). Basal respiration is altered in response to ATP demand and different substrates in the incubation medium. In our case, there are no changes or differences in incubation medium and therefore, infection condition is the only variable influencing basal respiration. As shown in Fig. 5C,F there is an increase in both proton leak OCR and ATP-production parameters in T. cruzi-infected cells. These effects could be due to oxidative stress induced by $T$. cruzi.

Energy phenotype results using Seahorse allowed evaluation of the capacity and the preferred energy supply pathway in AC16 and SGHPL-5 cells infected and non-infected with T. cruzi. Infection by T. cruzi induces mitochondrial activation in the cell, both in AC16 cardiomyocytes (Fig. 6A) and in SGHPL-5 trophoblastic cells (Fig. 6B). The effects after infection are similar in both cell lines. Infection with $T$. cruzi produced an increase of the mitochondrial respiration without considerable increases in the glycolytic flux. 


\section{DISCUSSION}

Understanding the cellular metabolic perturbations present in Chagas disease will expand the available therapeutic targets. Trypanosoma cruzi has various mechanisms to induce pathogenesis according to the infected tissue (Bonney and Engman 2008, Nagajyothi et al. 2011). In myocardial tissue an inflammatory response is observed after infection with $T$. cruzi. In adipose tissue T. cruzi influences the level of adipokines released, alters glucose metabolism and seems to act as a reservoir for the parasite (Combs et al. 2005).

Different reports suggest a key role of oxidative stress for Chagas disease progression (Gupta et al. 2009, Paiva et al. 2018). However, it is still unknown, and debated, if radical oxygen species act as a mechanism to eliminate the parasite, or whether oxidative stress promotes parasite infection depending on its concentration (Finzi et al. 2004, Paiva and Bozza 2014). In addition, there are many immunologic (Roggero et al. 2006), genetic and metabolic (Caradonna et al. 2013) endogenous host factors that modulate the different responses and progression rate of infection with $T$. cruzi, including polymorphisms in the major histocompatibility complex and cytokine genes involved in the innate and specific immune response (Ayo et al. 2013) and metabolic signalling and products of host cells.

The multifactorial cellular effects of $T$. cruzi make it difficult to standardise a unique methodology to evaluate cellular functions in Chagas disease. In our laboratory, by using a Seahorse XFe24 platform, we have established an efficient methodology to study the effects of immunological mediators of the host immune response and to test the effect of new drugs in diverse cell lines including cardiomyocytes and first trimester trophoblasts infected with $T$. cruzi. Using two cell lines relevant for the study of Chagas disease in vitro, we have described the step and protocol of infection with $T$. cruzi for mitochondrial bioenergetics assays to provide a comparable procedure to investigate mitochondrial function and general cellular metabolic parameters in adherent infected cells.

In a previous study where cell respiration was evaluated in neonatal cardiomyocytes infected with $T$. cruzi, the authors reported that the best cell density to seed cardiomyocytes in 24-well Seahorse cell culture microplates was $5 \times 10^{4}$ cells per well, similar to the cell seeding density we proposed here (Dias et al. 2017). No reports about mitochondrial function assessment in SGHPL-5 cells infected with T. cruzi using the Seahorse XFe24 platform have been published yet.

It is important to highlight that after exposure of the adherent cells to trypomastigotes of $T$. cruzi enough washing steps should be performed to obtain a clean cell monolayer in the Seahorse microplates. This ensures stable and validated readings derived from infected cells and not from remaining parasites in suspension. Previous work evaluating the bioenergetic profile of T. cruzi at its different stages revealed that trypomastigote suspensions cause unstable OCR and ECAR readings and that mitochondrial function parameters are different between stages of $T$. cruzi (see Shah-Simpson et al. 2016). We conclude that the protocol described here for assays in adherent cells infected with $T$. cruzi using the Seahorse XFe24 flux analyser is suitable for evaluating changes in the bioenergetic function of target cells to aid understanding of the physiopathology mechanisms of Chagas disease.

Acknowledgements. We thank members of the Traslational Biomedical Research Group. For the execution of this project, we received financial support from the National Financing Fund for Science, Technology and Innovation of Colombia "Francisco José de Caldas" - COLCIENCIAS Grant: CT-649-2014 Project Code: 656671240824 . This work was developed within the framework of the postdoctoral research training No. 784-2017, Grant: FP44842-152-2018.

\section{REFERENCES}

Andrade D., Serra R., Svensjo E., Lima A.P., Ramos E.S., Jr., Fortes F.S., Morandini A.C., Morandi V., Soeiro Mde N., Tanowitz H.B., Scharfstein J. 2012: Trypanosoma cruzi invades host cells through the activation of endothelin and bradykinin receptors: a converging pathway leading to chagasic vasculopathy. Br. J. Pharmacol. 165: 1333-1347.

Aridgides D., Salvador R., PereiraPerrin M. 2013: Trypanosoma cruzi coaxes cardiac fibroblasts into preventing cardiomyocyte death by activating nerve growth factor receptor TrkA. PLOS ONE 8: e57450.

Ayo C.M., Dalalio M.M., Visentainer J.E., Reis P.G., Sippert E.A., Jarduli L.R., Alves H.V., Sell A.M. 2013: Genetic susceptibility to Chagas disease: an overview about the infection and about the association between disease and the immune response genes. Biomed. Res. Int. 2013: 284729.

Ba X., Gupta S., Davidson M., Garg N.J. 2010: Trypanosoma cruzi induces the reactive oxygen species-PARP-1-RelA pathway for up-regulation of cytokine expression in cardiomyocytes. J. Biol. Chem. 285: 11596-11606.

Benirschke K., Kaufmann, P., Baergen R.N. 2006: Pathology of the Human Placenta, Fifth Edition. Springer-Verlag New York, 1,050 pp.
Bonney K.M., Engman D.M. 2008: Chagas heart disease pathogenesis: one mechanism or many? Curr. Mol. Med. 8: 510-518.

Caradonna K.L., Engel J.C., Jacobi D., Lee C.H., Burleigh B.A. 2013: Host metabolism regulates intracellular growth of Trypanosoma cruzi. Cell. Host. Microbe. 13: 108-117.

Castillo C., Muñoz L., Carrillo I., Liempi A., Gallardo C., Galanti N., Maya J.D., Kemmerling U. 2017: Ex vivo infection of human placental chorionic villi explants with Trypanosoma cruzi and Toxoplasma gondii induces different Toll-like receptor expression and cytokine/chemokine profiles. Am. J. Reprod. Immunol. 78: e12660.

Choy M.Y., St Whitley G., Manyonda I.T. 2000: Efficient, rapid and reliable establishment of human trophoblast cell lines using poly-L-ornithine. Early Pregnancy 4: 124-143.

Combs T.P., Nagajyothi, Mukherjee S., de Almeida C.J., Jelicks L.A., Schubert W., Lin Y., Jayabalan D.S., Zhao D., Braunstein V.L., Landskroner-Eiger S., Cordero A., FaCtor S.M., Weiss L.M., Lisanti M.P., Tanowitz H.B., SCHERER P.E. 2005: The adipocyte as an important target cell for Trypanosoma cruzi infection. J. Biol. Chem. 280: 2408524094. 
Coura J.R., Vinas P.A. 2010: Chagas disease: a new worldwide challenge. Nature 465: S6-7.

Cucunuba Z.M., Florez A.C., Cardenas A., Pavia P., Montilla M., Aldana R., Villamizar K., Rios L.C., Nicholls R.S., Puerta C.J. 2012: Prevalence and risk factors for Chagas disease in pregnant women in Casanare, Colombia. Am. J. Trop. Med. Hyg. 87: 837-842.

Dias P.P., Capila R.F., do Couto N.F., Estrada D., Gadelha F.R., Radi R., Piacenza L., Andrade L.O. 2017: Cardiomyocyte oxidants production may signal to $T$. cruzi intracellular development. PLoS Negl. Trop. Dis. 11: e0005852.

Diaz-Lujan C., Triquell M.F., Castillo C., Hardisson D., Kemmerling U., Fretes R.E. 2016: Role of placental barrier integrity in infection by Trypanosoma cruzi. Acta Trop. 164 360-368.

Duran-Rehbein G.A., Vargas-Zambrano J.C., Cuellar A., Puerta C.J., Gonzalez J.M. 2014: Mammalian cellular culture models of Trypanosoma cruzi infection: a review of the published literature. Parasite 21: 38 .

Finzi J.K., Chiavegatto C.W., Corat K.F., Lopez J.A., Cabrera O.G., Mielniczki-Pereira A.A., Colli W., Alves M.J., Gadelha F.R. 2004: Trypanosoma cruzi response to the oxidative stress generated by hydrogen peroxide. Mol. Biochem. Parasitol. 133: 37-43.

Gude N.M., Roberts C.T., Kalionis B., King R.G. 2004: Growth and function of the normal human placenta. Thromb. Res. 114: 397-407.

Gupta S., Wen J., Garg N.J. 2009: Oxidative stress in Chagas disease. Interdiscip. Perspect. Infect. Dis. 2009: 190354.

Kipkeew F., Kirsch M., Klein D., Wuelling M., WinterhaGer E., Gellhaus A. 2016: CCN1 (CYR61) and CCN3 (NOV) signaling drives human trophoblast cells into senescence and stimulates migration properties. Cell. Adh. Migr. 10: 163-178.

Liempi A., Castillo C., Duaso J., Droguett D., Sandoval A., Barahona K., Hernandez A., Galanti N., Maya J.D., Kemmerling U. 2014: Trypanosoma cruzi induces trophoblast differentiation: a potential local antiparasitic mechanism of the human placenta? Placenta 35: 1035-1042.

Nagajyothi F., Weiss L.M., Silver D.L., Desruisseaux M.S., Scherer P.E., Herz J., Tanowitz H.B. 2011: Trypanosoma cruzi utilizes the host low density lipoprotein receptor in invasion. PLoS. Negl. Trop. Dis. 5: e953.

Paiva C.N., Bozza M.T. 2014: Are reactive oxygen species always detrimental to pathogens? Antioxid. Redox. Signal 20: $1000-1037$.

Paiva C.N., Medei E., Bozza M.T. 2018: ROS and Trypanosoma cruzi: Fuel to infection, poison to the heart. PLoS Pathog. 14: e1006928.

Roggero E., Perez A.R., Tamae-Kakazu M., Piazzon I., Nepomnaschy I., Besedovsky H.O., Bottasso O.A., DeL REY A. 2006: Endogenous glucocorticoids cause thymus atrophy but are protective during acute Trypanosoma cruzi infection. J. Endocrinol. 190: 495-503.

Shah-Simpson S., Pereira C.F., Dumoulin P.C., Caradonna K.L., Burleigh B.A. 2016: Bioenergetic profiling of Trypanosoma cruzi life stages using Seahorse extracellular flux technology. Mol. Biochem. Parasitol. 208: 91-95.

Wen J.J., Yin Y.W., GaRG N.J. 2018: PARP1 depletion improves mitochondrial and heart function in Chagas disease: effects on POLG dependent mtDNA maintenance. PLoS Pathog. 14: e1007065.

Cite this article as: González-Ortiz L.M., Sánchez-Villamil J.P., Celis-Rodríguez M.A., Lineros G., Sanabria-Barrera S., Serrano N.C., Rincon M.Y., Bautista-Niño P.K. Measuring mitochondrial respiration in adherent cells infected with Trypanosoma cruzi Chagas, 1909 using Seahorse extracellular flux analyser. Folia Parasitol. 66: 016. 